\title{
Evaluation of routing protocol with multi-mobile sinks in WSNs using QoS and energy consumption parameters
}

\author{
Nadia A. Shiltagh', Mahmood Z. Abdullah², Ahmed R. Zarzoor ${ }^{3}$ \\ ${ }^{1}$ College of Engineering, University of Baghdad, Baghdad, Iraq \\ ${ }^{2}$ College of Engineering, Mustansiriyah University, Baghdad, Iraq \\ ${ }^{3}$ Institute for Post-graduation Studies Iraqi Commission for Computer and informatics, Baghdad, Iraq
}

\begin{tabular}{l}
\hline Article Info \\
\hline Article history: \\
Received Sep 20, 2018 \\
Revised Mar 23, 2019 \\
Accepted Apr 1, 2019 \\
\hline
\end{tabular}

\section{Keywords:}

Base station (BS)

Group of mobile sinks (GMSs)

Anchor nodes (ANs)

Quality of services (QoS)

Reference node (RN)

Sink node (SN)

Wireless sensor networks

(WSNs)

\begin{abstract}
An efficient networks' energy consumption and Quality of Services (QoS) are considered the most important issues, to evaluate the route quality of the designed routing protocol in Wireless Sensor Networks (WSNs). This study is presented an evaluation performance technique to evaluate two routing protocols: Secure for Mobile Sink Node location using Dynamic Routing Protocol (SMSNDRP) and routing protocol that used K-means algorithm to form Data Gathered Path (KM-DGP), on small and large network with Group of Mobile Sinks (GMSs). The propose technique is based on QoS and sensor nodes' energy consumption parameters to assess route quality and networks' energy usage. The evaluation technique is conducted on two routing protocols in two phases: The first phase is used to evaluate the route quality and networks' energy consumption on small WSN with one mobile Sink Node (SN) and GMSs. The second phase, is used to evaluate the route quality and networks' energy consumption on large network (four WSNs) with GMSs. The two phases are implementated by creating five sceneries via using NS2.3 simulator software. The implementation results of the proposed performance evaluation technique have demonstrated that SMSNDRP gives better networks' energy consumption on small single network in comparison with KM-DGP. Also, it gives high quality route in large network that used four mobile SN, in contrast to KM-DGP that used sixteen mobile SNs. While in large network, it found that KM-DGP with sixteen mobile SNs gives better networks' energy consumption in comparison with SMSNDRP with four mobile SNs.
\end{abstract}

Copyright @ 2019 Institute of Advanced Engineering and Science. All rights reserved.

Corresponding Author:

Ahmed R. Zarzoor, Institute for Post-graduation Studies, Iraqi Commission for Computer \& informatics, Baghdad, Iraq.

Email: Ahmed.Arjabi@gmail.com

\section{INTRODUCTION}

The large network is composed of a number of WSNs, each of a WSN is consisted of a lot number of sensor nodes that deployed in wide geographical area. These sensor nodes would gathered data from their environment and forwarded it to the SN via multiple hops [1]. Which in turn would exhausted sensor nodes' remaining energy and reduced the entire network lifetime. Consequently, designing routing protocol efficiently, is considered a big challenge to the researchers, due to number of issues: the first one, is that all the sensor nodes which are closed to the SN location would lost their energy very fast (i.e. died), since they conveyed data of other sensor nodes in addition to their own data. So, when they are died this would in turn create a hole near the $\mathrm{SN}$, which caused a hot spot energy problem [2]. To overcome this problem, researchers are suggested to use a mobile $\mathrm{SN}$ instead of a static $\mathrm{SN}$, in order to balance the energy usage on 
different areas in the WSN [3, 4] and mitigate the hot spot energy problem [5]. The second issue, is about considering the QoS constratians when evaluaton of the routing path quality [6,7], i.e. the best path quality is the one that achieved the lowest (delay and total data dropped packets) and gives highest (Packet Deliveary Ratio (PDR) and Average throughput) from other designed paths in the network. Thus, using a small number of mobile SN in a large WSN is considered impractical solution, because it maximized latency. To overcome this problem, one of the solation is to decease the latency by dividing the large network area into sub-areas and using a GMSs [8]. Finally, the third issue, is about considering the efficienty of the designed routing paths between each of the mobile SN and BS, by taking into account two things: The first one, is the way that the sensor nodes are deployed in the network because there is some sub-area with high density, while other with low-density. In the high-density area, a lot of data transmission processing would occurred frequently. Besides, route reliability is increased because there is more than one route can be constructed among any pair of sensor nodes in the network. While in the low-density, data conveyed process and route reliability is decreased. The second one, when using a larger number of mobile SNs this would increased the network cost and maximized the overhead complexity to maintain routing table for each $\mathrm{SN}$ in the network.

Therefore, this study is aimed to propose a new evaluation method of the routing protocol that would cover the three menstion above issues. In order, to evaluate the performance of "Secure Mobile Sink Node location using Dynamic Routing Protocol" (SMSNDRP) that was developed by us in the previous studies [9, 10], via making a comparsion of its performance in small and large network with other routing protocol called K-means to form Data Gathered Path (KM-DGP) which is described in related works section. So, the evaluation process is conducaded in two phases: small WSN (with one mbile SN and GMSs) and Group of WSNs with GMSs based on QoS and the sensor nodes' energy consumption parameters. However, the performance results of SMSNDRP in small WSN gives high qulity of routing routes and consumed less energy in comparsion with the KM-DGP. While, in large network the KM-DGP is consumed less energy and gives low qulity routing routes in comparsion with the SMSNDRP. The rest of this study will be presented as follows: section 2 explored of related works that used routing protocol on WSNs with GMSs, Section 3 described performance evaluation method two phases: evaluation of Route quality for small WSN and evaluation route quality for large network. Section 4, discussed the implementation results of five scenarios: the first three scenarios are used in small WSN that consisted of 50 nodes with one or four mobile SN. The last two scenarios are used in large network that composed of 200 nodes, four WSNs, four or sixteen mobile SN, four or sixteen reference nodes, six anchor nodes and single BS. Finally, section 5 includes study conclusion.

\section{RELATED WORKS}

There are a number of studies that proposed designing a route with the GMSs. This was considered due the GMSs' route having a major benefit, since they reduced data traffic around the SN and alleviate the hot spot-energy problem. In Farooq et. al study, they proposed a technique called "Low-Power and Lossy Networks" RPL [11]. In this technique they solved high data traffic jam via selected the optimal path, that is used for routing data from sensor node to the GMSs with minimum number of hop, lowest latency and maximum PDR. The path selection is based on number of parameters such as the size of remaining data on communication link (large size means more bandwidth available on the link), the waiting time for each packet in "MAC layer queue" [11] before it conveyed to the next destination node (i.e. latency), managing data packet in MAC layer buffer or queue occupancy (e.g. place the packets that come from the node with high rate generated first in the queue) and hop counter. Thus, the path that provided high bandwidth, lowest delay and required minimum number of hop will be selected as the optimal path for routing data in the network.

In Chatterjee and Das [12] study, they proposed a method to deploy GMSs in a large network based on graphic theorem. This was proposed in order to optimize the number of clusters and cluster coverage range. To illustrate graphic theorem, let say graphic $\mathrm{G}(\mathrm{N}, \mathrm{E})$, where $\mathrm{N}$ is the number of nodes' neighbor and $\mathrm{E}$ is the number of edges that were used to connect the current node with its' neighbors in one cluster. The Euclidean distance is used to calculate the distance between the current node and its' neighbors, which represent the hop-distance (i.e. number of hop) on the short path between them. The clusters are constructed after the GMSs and sensor nodes are deployed randomly in the area, then a random selection for seed nodes is made, in order to form the clusters based on using $\mathrm{G}(\mathrm{N}, \mathrm{E})$ and hop-distance. In Barani et. Al study [13], they investigated the effects of mobile sink position on the network capacity and latency parameters. They used two different SN position schemes: the common position scheme and random scheme. They studied the two schemes via deployed SNs on number of cells either randomly or based on the common location rule (the most visited locations on the cells by SN in previously). In the next step, each sensor node would searched to find the SN in one hop to transmit its' sensory data to this SN, if there is no SN in one hop 
then it would searched for rely node, which in turn repeat the same process until it the SN location is reached. Also, they used redundant packets with SNs' position, in order to measure its impact on the network capacity and latency parameters. They found it has no impact on capacity and its reduced latency. Although in [14] study, the researchers suggest a method to decrease the hop-distance that was required to convey data in a trip from the sensor node to the SN. In the first step of their method, they divided the network into a number of isolated clusters using K-mean algorithm. In the next step they deployed GMSs using "Particle Swarm Optimization" PSO algorithm.

In another study [15], researchers improved the network lifetime via using GMSs with WSN. They proposed a technique, in which the SN is moved toward high density area in the network. In this area, each sensor node is connected to the mobile SN through one-hop only. So, the far sensor nodes that are not in the coverage range of SN may be covered by other deployed SNs' coverage range. The main idea is to make each sensor node use one-hop to convey its' data to the SN. While Singh et al, proposed using a virtual grid cells to propagate data between the source "dissemination nodes" and mobile SN [16]. To illustrate, if GMSs are deployed in area and BS send a query for data in that area, then all the sensor nodes in that area will cooperated together to construct a "virtual grid cell". The sized of each cell in that grid is dependent on sensor nodes' radius size. The next step is about making a selection for the dissemination node, which is the node that has the highest residual energy and responsible for query transmission to the SN via using short path. The SN in turn can estimates the distance and specified the sensor nodes' location in that area based on the received query. Subsequently, the best path to that area is specified and SN can move toward it. While Wang et al, proposed a method that used clustering technique with GMSs, in order to improve the network lifetime [17]. They divided the large network into several clusters via using LEACH protocol and the used "Ant Colony Optimization" to find the best mobility path for the mobile SN. In order to collect data from Cluster Head $\mathrm{CH}$ of each cluster in the network.

In [18] the researchers proposed group of mobile SNs with routing protocol in underwater WSNs to maximize PDR and minimize the networks' energy exhaustion. This protocol consisted of two phases: layering and construction. In the layering phase, the sensor nodes are deployed around the SN in (n) layers. The SN is allocated in layer (0), sensor nodes with one-hop from SN are allocated in layer (1), with 2-hop are placed in layer (2) and so on. The sensor nodes are re-deployed periodically, when PDR become below the threshold value, due to SN mobility. In construction phase, sensor nodes in layer (1) send the data direct to the $\mathrm{SN}$, while sensor nodes in layer $2,3 \ldots, \mathrm{n}$ would selected as relay node, inorder to forward data to next layer until SN is reached. Although Uppal et al, proposed using GMSs and multi-path technique with WSNs, in order to avoid link communication failures and reduced energy consumption in the network [19]. The main idea in their study is about using routing table that is formed by each sensor node in the network. The routing table would continued parameters such as number of hops, successful conveyed data ratio and remaining energy. So, each sensor node in the network established multi-path with its' neighbour via distance (onehop). Thus, the optimal path is path that has minimum number of hops, constricted from sensor nodes' that have high residual energy and maximum data conveyed ratio.

Also, Gebremeskel and Rao [20], used GMSs to improve network lifetime based on changing the SNs' location in the network. They distributed the SNs and sensor nodes randomly in the network then constracted clusters in the network by using K-means method. In this method the SNs will be selected randomly. The next step in this method is about calculating the distance between each sensor node and SN in the network. Consequently, each sensor node that would have less distance to the SN will join its' cluster. Finally, the SNs change their position and move toward the center point of each clusters. So, in this way network lifetime is optimized by decreasing the distance between SN and sensor node. In other study [21], Although they used K-means algorithm to split the large scale network into clusters and specified data gathering path (DGP), that used to move mobile SN in a way that optimized the network lifetime. Since, each data gather point on the routing path is the optimal point location in the cluster that specified by K-means algorithm. So, the mobile SN is stops on it for period of time to collect data and then move toward the next point in the DGP.

However, most of the above studies used GMSs, in order to solve the problem of hot spot energy problem and prolong the network lifetime. But this solution still considered as high cost solution because of using more than one mobile SNs in large network. Therefore, in this study an evaluation method is proposed to evaluate the performance of SMSNDRP that used a small number of mobile SNs in small WSN and large network based on QoS and sensor nodes' energy consumpation parameters.

\section{PERFORMANCE EVALUATION METHOD}

In this paper an evaluation performance technique is proposed to measure the route quality and energy usage in large and small network based on QoS parameters (End to End delay, Average throughput, 
PDR and total number of dropped data packets) that are used to evaluate the route quality of two routing protocols (SMSNDRP and KM-DGP). Also, the method based on three of sensor nodes' energy consumption parameters which are: number of nodes with sufficient energy, number of died nodes and number of nodes with low energy. So, the best networks' energy consumption is achieved when there is low number of died nodes and high number of nodes with sufficient energy during routing data process in the network. However, the evaluation performance method is condaucted into two phases:

a. Evaluation of Routes Quality for Small Network (Single WSN)

b. Evaluation of Routes Quality for Large Network (Four WSNs)

\subsection{Evaluation of routes quality for small network (single WSN)}

In this phase, an evaluation is conducted to the route quality and sensor nodes' energy consumption on small single WSN, via using two routing protocols: SMSNDRP and KM-DGP. The routing protocol SMSNDRP is aimed to move mobile SN in the network in a way that reduced network energy consumption, and maximized the anonymity of SNs' location against traffic analysis attack. However, in the first step of the SMSNDRP, is about dividing the network into number of sub-area in order to form clusters and elect CHs using DECAR [20] algorithm. So, the node is selected as $\mathrm{CH}$, if it has the highest remaining energy and minimum distance from SN in comparsion with other sensor nodes in the same cluster. The second step, is about calculating the coordination points $(\mathrm{x}, \mathrm{y})$ for the new SNs' location via computing the mean function of all elected CHs' location, in order to move SN toward it in the network. The third step, is about the forming the path between $\mathrm{CH}$ and mobile $\mathrm{SN}$, through rearranging $\mathrm{CHs}$ in ascending order according to their distance from SN and then rearrange them again according to their residual energy in descending order. So, the CHs with higher remaining energy will be placed in nearst position to the $\mathrm{SN}$ and only its will be allowed to send data directly to the SN. The routing path is reconstructing again through using the same steps that mention above, when one or more $\mathrm{CHs}$ ' residual energy is below the energy threshold value.

While in the KM-DGP, the first step is about forming clusters via random deployment of sensor nodes in the network. In the next step, the seed nodes are selected randomly and the distance between the seed node and each sensor node in the network is calculated. Thus, each sensor node that have minimum distance to the seed will be added to the cluster. The third step, is about computing the average nodes' coordination point value in each cluster. So, if there is no change in the cluster then stop otherwise repeat from step 2. At the end, the optimal routing path DGP is constructed from the last average coordination point value in each cluster. Subsequently, the SNs are moved in the network using DGP, in order to collect data from sensor nodes in each cluster in the network.

However, the evaluation process in this phase is implemented on single small WSN will be as follow:

a. Evaluate the energy consumption for the paths that generated by SMSNDRP and KM-DGP with single mobile sink node by using networks' energy consumption parameters

b. Evaluate the route quality for the paths that generated by SMSNDRP and KM-DGP with single mobile SN through using QoS parameters

c. Evaluate the energy consumption for the path that generated by KM-DGP with four mobile SNs via using networks' energy consumption parameters

d. Evaluate the route quality for the paths that generated by KM-DGP with four mobile SNs via using QoS parameters

\subsection{Evaluation of routes quality for large network (four WSNs)}

In this phase, the same QoS and sensor nodes' energy consumption parameters that used in phase one will be used in this phase for large network (four WSNs). Also, the best path quality between each SN and BS, is designed via using method that developed by us in study [10]. In this method the optimal path is designed based on six anchor nodes and one reference node for each mobile SN in each WSN. The six anchor nodes are formed a ring around unknown BS location in order to specify it location. While, the reference node is used to gather the new SN location infomation in each WSN and send it to the anchor nodes, which in turn store mobile SN location information in routing table that maintained by anchor node. The routing table contained: WSN ID, number of hops, SN location, BS location and path details. Thus, according to routing table information the best route is selected between mobile SN and BS. The best route is the one that has minimum number of hops and consumed less sensor nodes' residual energy. Although, its quality is evaluated based on QoS parameters.

So, the evaluation process in this phase is implemented on large network will be as follow:

1. Evaluate the energy consumption for paths that generated into two large networks: one used SMSNDRP with four mobile SNs and the other one is used KM-DGP with sixteen mobile SNs. Also, the optimal path between SN and BS in both network is designed via using anchor nodes and reference node. The network 
energy consumption is computed through using the same sensor nodes' energy parameter that used in phase one.

2. Evaluate the route quality for the paths that generated in same two large networks in step one. The route quality is computed through using the same QoS parameters that used in phase one see Figure 1, which demonstrated the two phases of the proposed performance evaluation method in this paper.

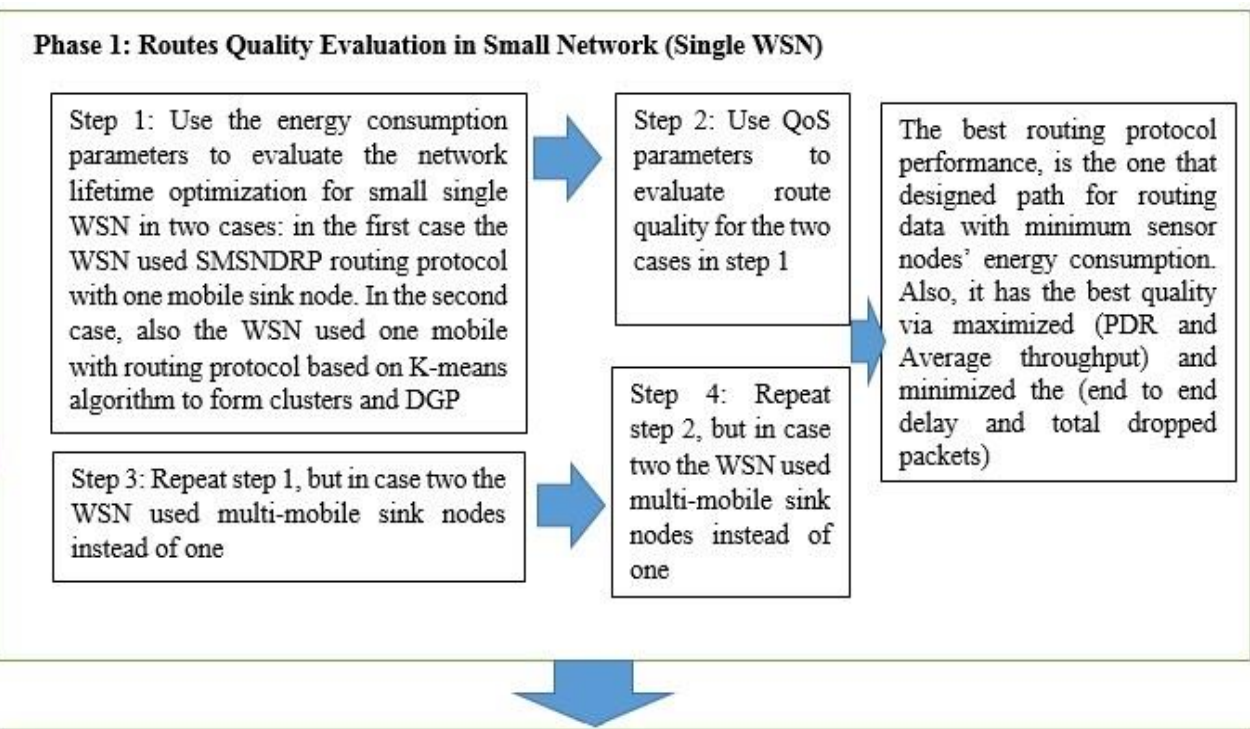

Phase 2: Routes Quality Evaluation in Large Network (more than two WSNs)

Step 1: Use the energy consumption parameters to evaluate the network lifetime optimization for all the paths between each mobile SN and BS in two large networks: one used KM-DGP and the other one used SMSNDRP with multi-mobile sinks nodes.

Step 2: Use the QoS parameters to evaluate the route quality for all the paths between each mobile sink node and base station in the two networks in step 1

The best routing protocol, is the one that designed path for routing data between (sensor nodes and sink node) and between (each sink node in each WSN and base station) with minimum sensor nodes' energy consumption. Also, it has the best quality via maximized (PDR and Average throughput) and minimized the (end to end delay and total dropped packets)

Figure 1. Illustrate the study method two phases

\section{RESULTS AND ANALYSIS}

The routing protocol SMSNDRP has been evaluated before in small network based on QoS parameters in our pervious study [22]. In this study a new evaluation performance method is applied on SMSNDRP to evaluate it performances in small and large network, based on QoS and the sensor nodes' energy consumption. Also, a comparsion with other routing protovol KM-DGP has been done in this study, in order to analysis its performance. So, the evaluation process is conducaded via cearting five scenarios using NS2.3 software simulator. The first three scenarios are conducted on single WSN that consisted of 50 nodes, one mobile SN for scenario 1 and 2 and four mobile SNs for scenario 3. While the last two scenarios are conducted on large network consisted of four WSNs, 200 nodes, six anchor nodes, four reference nodes for scenario 4, sixteen reference nodes for scenario 5, and one BS for scenario 4 and 5 , see Table 1 . So, the first phase of the study method is implemented in scenario 1,2 and 3 , while the second phase is performed in scenario 4 and 5. 
Table 1. Simulation parameters details

\begin{tabular}{cc}
\hline Parameters & Values \\
\hline Time of simulation end & $60 \mathrm{~s}$ \\
Number of nodes & 50 for scenario 1,2 and 3 \\
& 200 for scenario 4 and 5 \\
Number of sink nodes & 1 for scenario 1 and 2 \\
& 4 for scenario 3 and 4 \\
& 16 for scenario 5 \\
Number of anchor nodes & Not used for scenario 1,2 and 3 \\
& 6 for scenario 4 and 5 \\
Number of reference node & Not used scenario for 1,2 and 3 \\
& 4 for scenario 4 \\
Channel type & 16 for scenario 5 \\
Network interface type & Channel/WirelessChannel \\
Area & Phy/WirelessPy \\
MAC type & $1000 x 1000$ \\
Traffic Model & Mac/802_11 \\
& FTP
\end{tabular}

\subsection{Small network implementation results}

In scenario 1, the routing protocol SMSNDRP is implemented on a WSN that consisted of 50 nodes with single SN, see Figure 2. The simulation result for sensor nodes' energy consumption parameters are: number of nodes with sufficient energy is 25, number of nodes with low energy is 24 and number of die nodes is 1. For QoS parameters the results are: PDR is $99.261 \%$, Total dropped packet is 10, Average Throughput is $134.55 \mathrm{kbps}$ and End to End latency is 328.4652 milliseconds. Also, in scenario 2 the same WSN structured that used in the first scenario will be used, but using routing protocol KM-DGP instead of SMSNDRP, see Figure 3. The simulation result for sensor nodes' energy consumption parameters are: number of nodes with sufficient energy is 20, number of nodes with low energy is 13 and number of die nodes is 17. For QoS parameters the result: PDR is $98.261 \%$, Total dropped packet is 54, Average Throughput is $308.02 \mathrm{kbps}$ and End to End latency is 147.688 milliseconds.

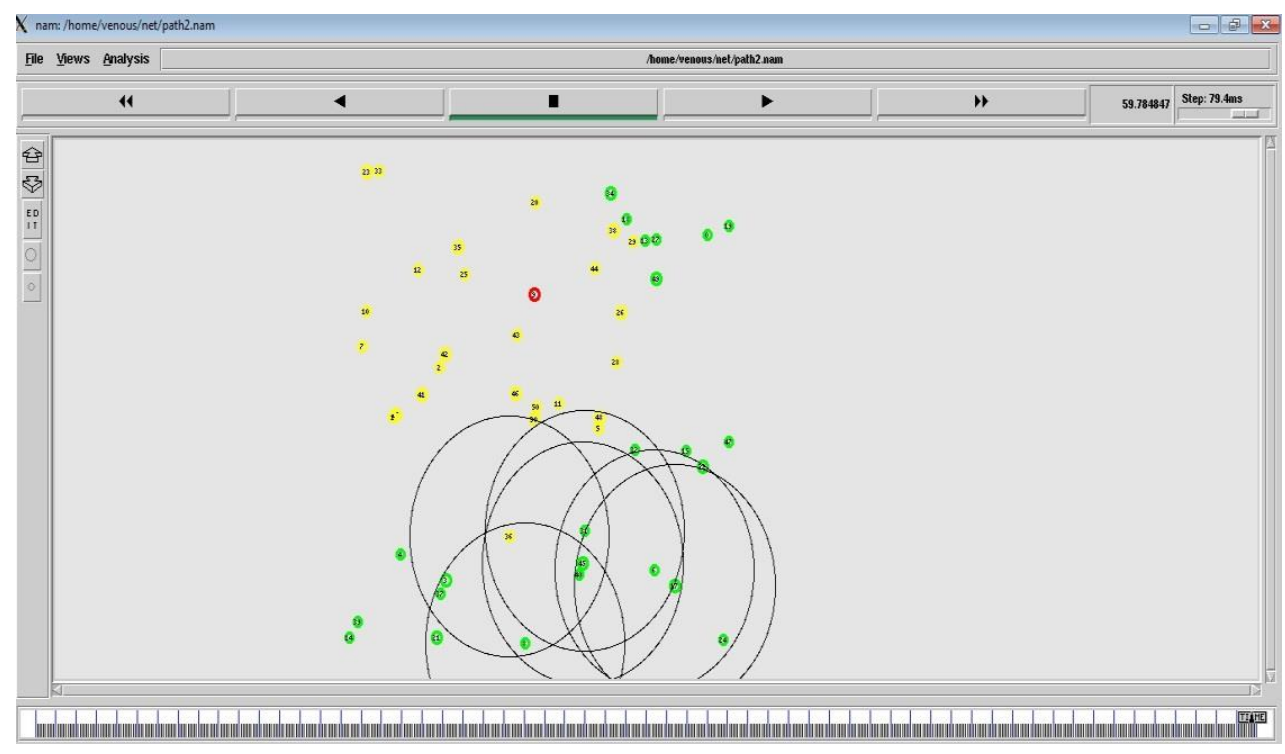

Figure 2. Scenario 1 screenshot from NS2.3 simulator software, that demonstrated the implementation of routing protocol SMSNDRP with one mobile SN in the network. The green node represents node with sufficient energy, yellow node represents node with low energy and red node represent the die nodes

In scenario 3 the same WSN structure of the second scenario is used again, but with four mobile SNs, see Figure 4. The simulation result for sensor nodes' energy consumption parameters are: number of nodes with sufficient energy is 4 , number of nodes with low energy is 4 and number of die nodes is 42 . For QoS parameters the result: PDR is $97.611 \%$, Total dropped packet is 100, Average Throughput is $419.02 \mathrm{kbps}$ and End to End latency is $\mathbf{1 7 4 . 6 8 8}$ milliseconds. However, based on the results, it has been 
discovered that SMSNDRP with one mobile SN improved the network energy consumption, in comparison with using routing protocol KM-DGP with one or four mobile SNs in small network, see Figure 5. Nevertheless, when comparison has made according to route quality using QoS parameters, it found the best route quality that is generated by SMSNDRP since its give the highest End to End latency (328.4652 milliseconds) value in comparison with KM-DGP with one mobile SN (147.688 milliseconds) value or four SNs (174.688 milliseconds) value, see Figure 6. But, its give the lowest data dropped packet (value=10) in comparison with KM-DGP that operate with one mobile SN (value=54) or four mobile SNs (value=100), for PDR the SMSNDRP gives the highest value (99.2614\%) in comparison with KM-DGP, see Figure 7. So, the route quality that is generated by the KM-DGP with four mobile SNs gives better performance than the route qulity that is generated by SMSNDRP with one mobile SN, because its minimized the latency and maximized average throughput. But still the routes that are generated by SMSNDRP with one mobile SN gives a better performance for PDR and total dropped packets in contrast with KM-DGP with one or four mobile SNs.

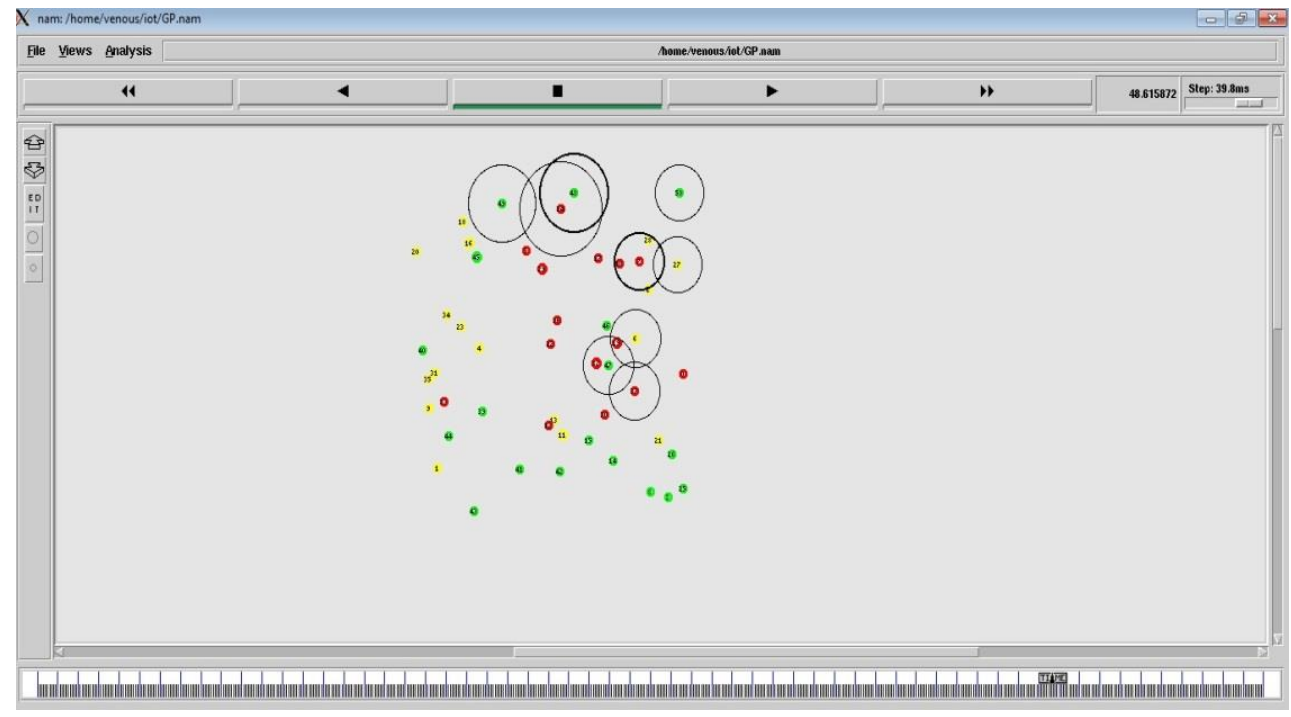

Figure 3. Scenario 2 screenshot from NS2.3 simulator software that demonstrated the implementation of routing protocol KM-DGP with one mobile $\mathrm{SN}$ in the network

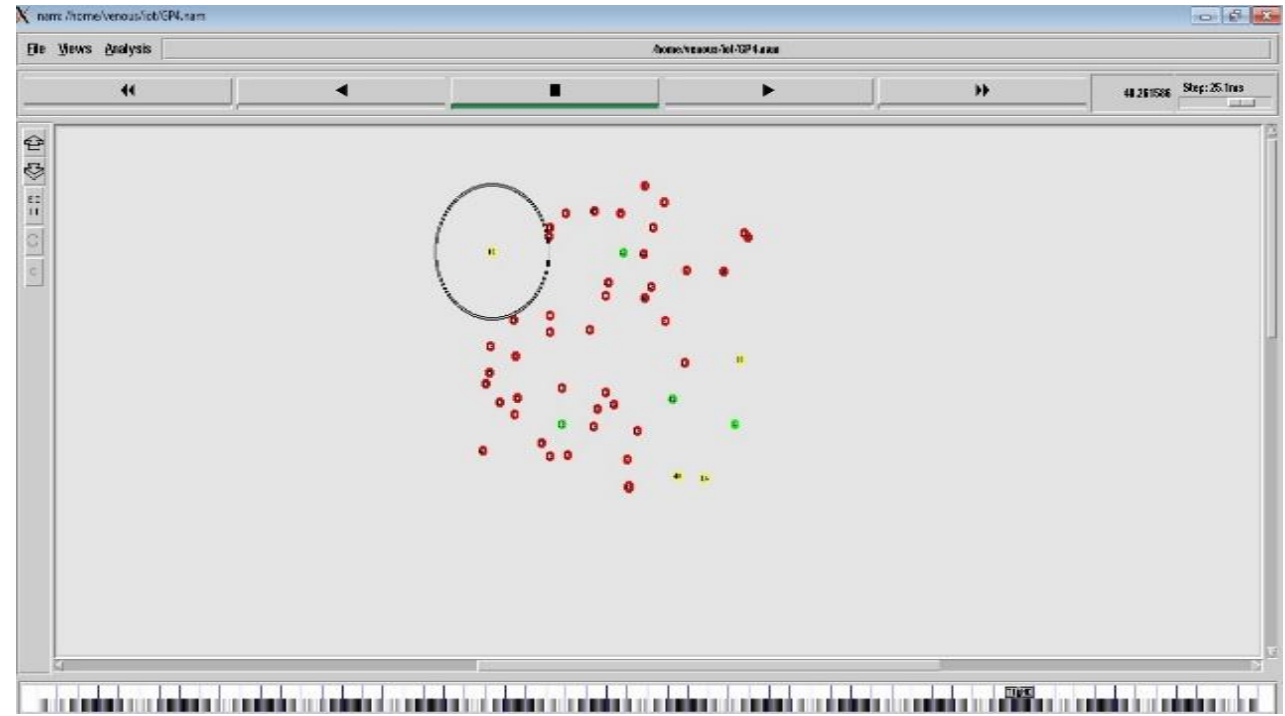

Figure 4. Scenario 3 screenshot from NS2.3 simulator software that demonstrated the implementation of routing protocol KM-DGP in the network with four mobile SNs 


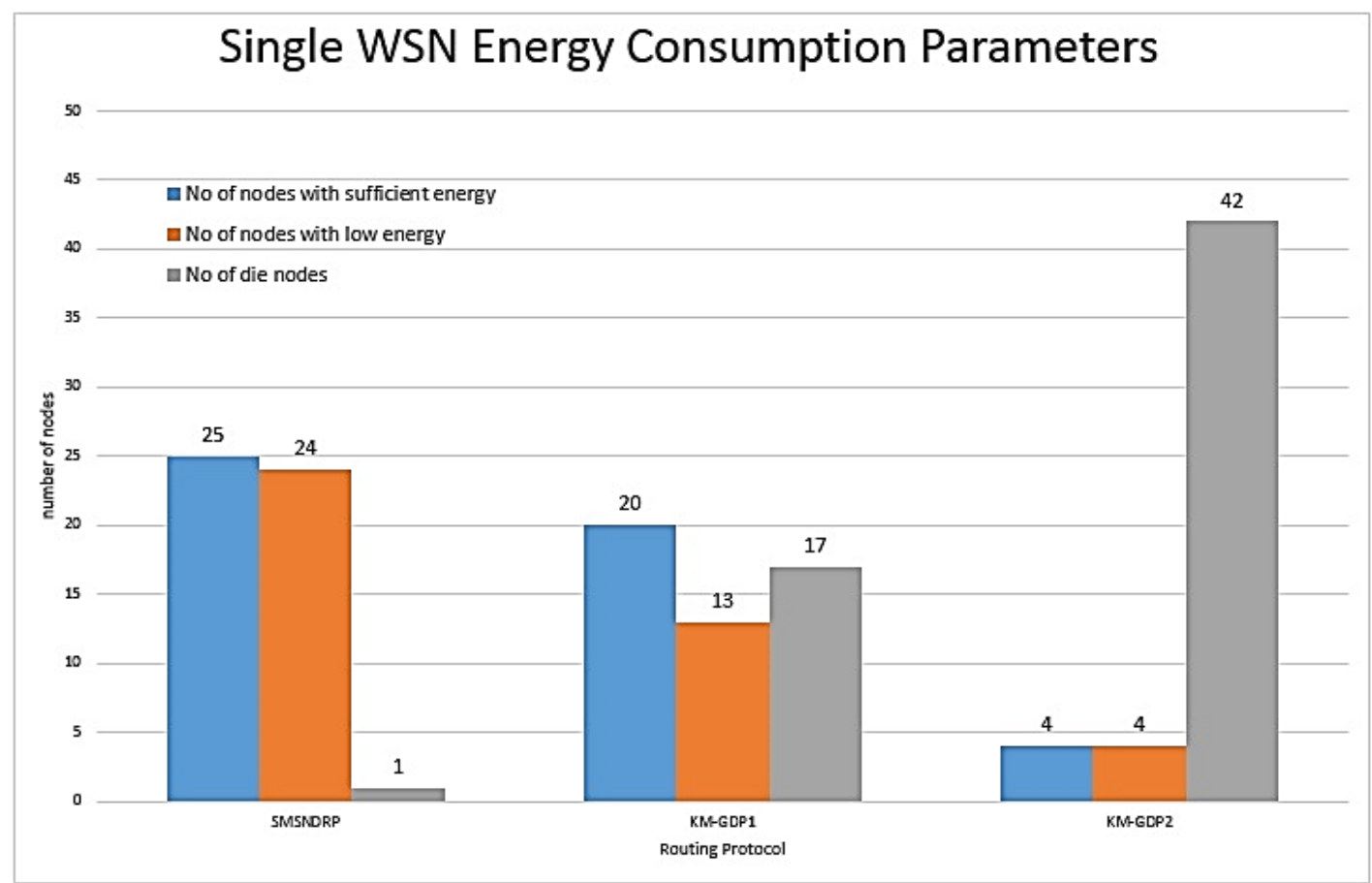

Figure 5. Demonstrate that SMSNDRP given better sensor nodes' energy consumption in comparison with KM-DGP, because it gives the lowest number of die nodes and maximum number of nodes with sufficient energy

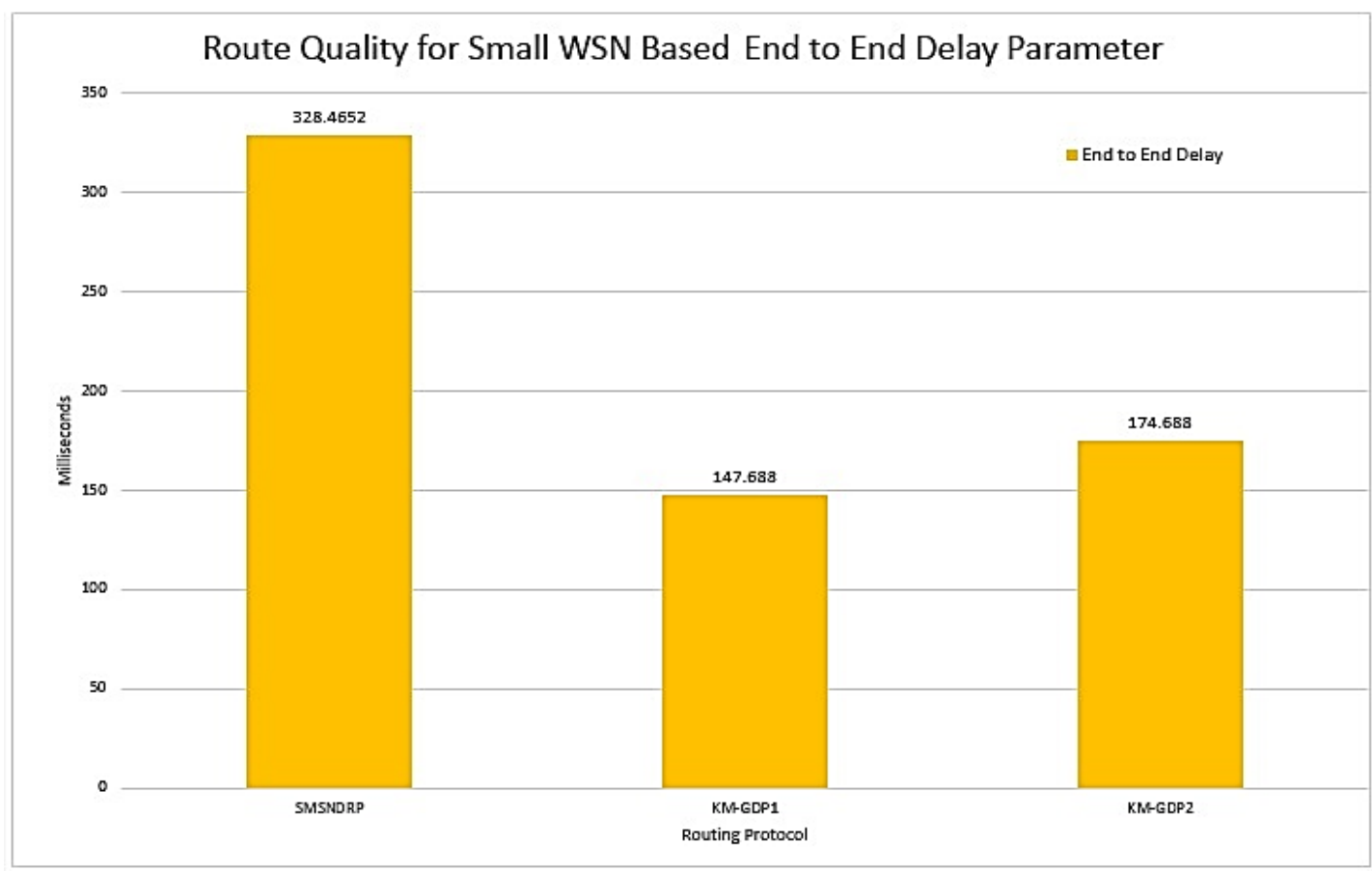

Figure 6. Demonstrate that KM-DGP produced the lowest end to end latency in comparison with SMSNDRP, which make it gives better network performance than SMSNDRP 


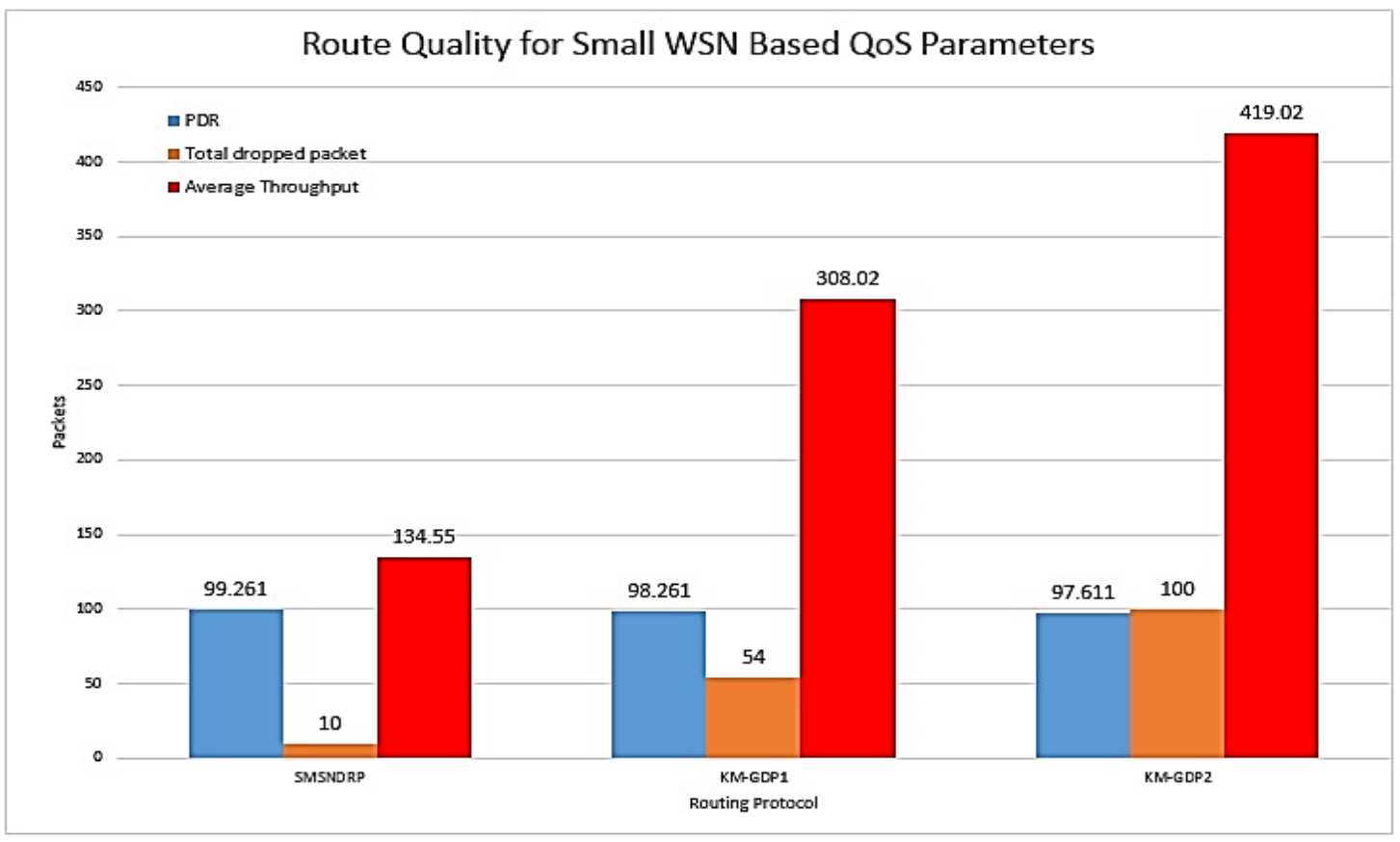

Figure 7. Demonstrate that SMSNDRP given the highest PDR value and lowest total dropped packets in comparison with KM-DGP, which make it gives better network performance than KM-DGP

\subsection{Large network implementation results}

The second phase of evaluation method is applied on large network using two scenarios 4 and 5 . In scenario 4 the routing protocol SMSNDRP is implemented on four WSNs, each one has single mobile SN and consisted of 50 nodes and one reference node for each mobile SN, see Figure 8 . The reference node stores the last location information that SN is moved toward it. Also, reference node sends SN new position in each WSN to the six anchor nodes, that form a ring around one BS The anchor nodes are used to specify the unknown BS location and construct the routing path between (each SN in each WSN and the BS) based on the routing table information [10]. The simulation result of scenario 4, for sensor nodes' energy consumption parameters as follows: number of nodes with sufficient energy is 128, number of nodes with low energy is 38 and number of die nodes is 34. For QoS parameters the result: PDR is 98.261\%, Total dropped packet is 336, Average Throughput is $1966 \mathrm{kbps}$ and End to End latency is 187 milliseconds. While, in scenario 5 the KM-DGP is used instead of SMSNDRP on the same WSN structure that used in scenario 4 but with sixteen mobile SNs, see Figure 9. The simulation result of scenario 4, for sensor nodes' energy consumption parameters as follows: number of node with sufficient energy is 164, number of nodes with low energy is 10 and number of die nodes is 26. For QoS parameters the result: PDR is 74.128\%, Total dropped packet is 1988, Average Throughput is $781.72 \mathrm{kbps}$ and End to End latency is 503 milliseconds.

So, based on the scenario 4 and 5 results, the routing protocol KM-DGP with 16 mobile SNs improved the network energy consumption, in comparison with the routing protocol SMSNDRP with four mobile SNs in large network, see Figure 10. Nevertheless, when made a comparison based on route quality using QoS parameters, it found the best route quality is the one that generated by SMSNDRP, because it gives the lowest End to End latency (187 milliseconds) value in comparison with KM-DGP (503 milliseconds), see Figure 11. Although, it gives the lowest data dropped packet (value=1966) in comparison with the KM-DGP (value=1998). Also, for PDR the SMSNDRP gives the highest (value=98.261\%) in comparison with KM-DGP (value=74.128\%). Thus, the route quality of the SMSNDRP with four mobile SNs is better than route quality that generated by KM-DGP with sixteen mobile SNs, because it minimized the (latency and total dropped packets) and maximized (average throughput and PDR), see Figure 12. 


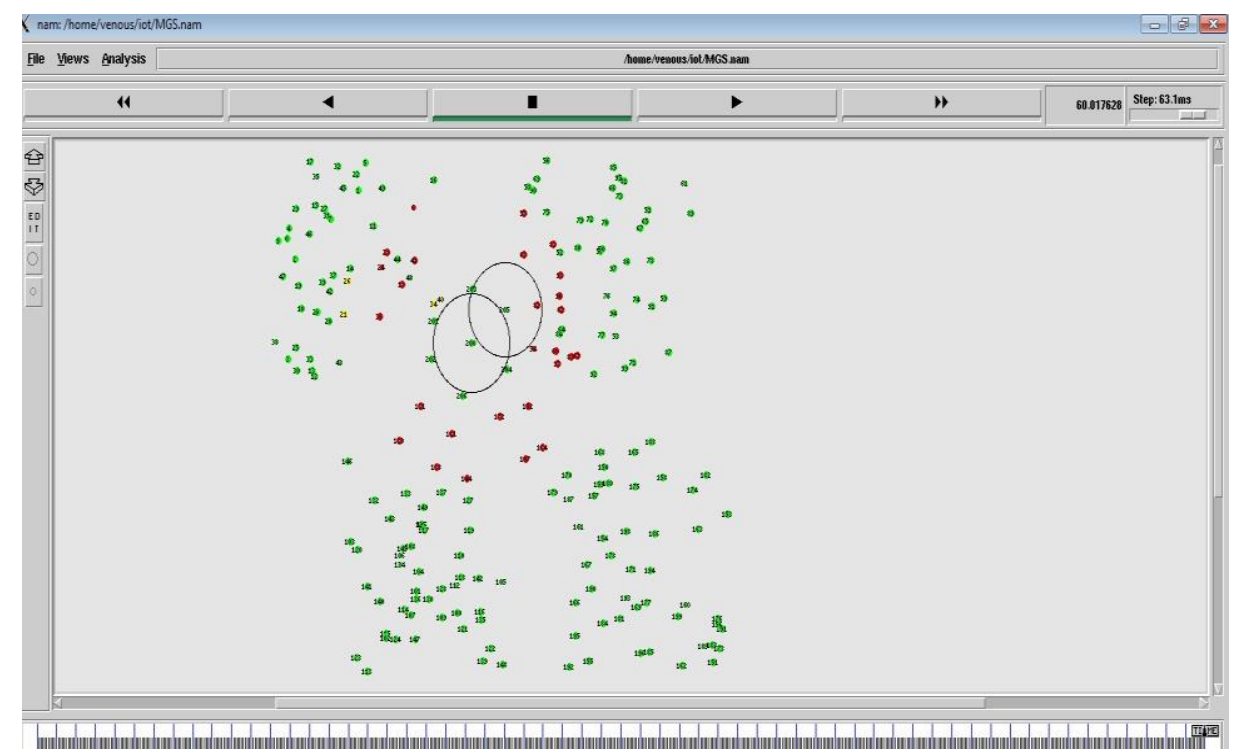

Figure 8. Scenario 4 screenshot from NS2.3 simulator software, that demonstrated the implementation of routing protocol SMSNDRP in the network with four WSNs and each has one mobile SNs, one reference node for each WSN, six anchor node and one BS

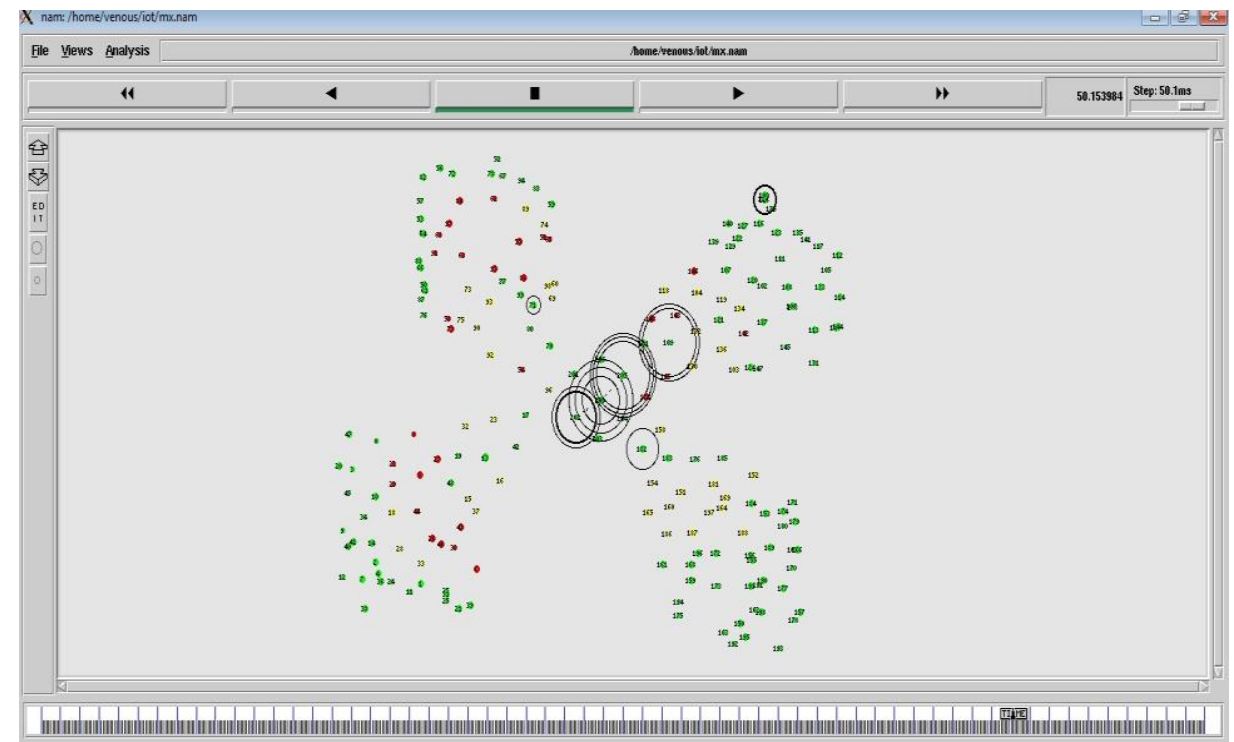

Figure 9. Scenario 5 screenshot from NS2.3 simulator software that demonstrated the implementation of routing protocol KM-DGP in the network with four WSNs and each has four mobile SNs, four reference node for each WSN, six anchor node and one BS 


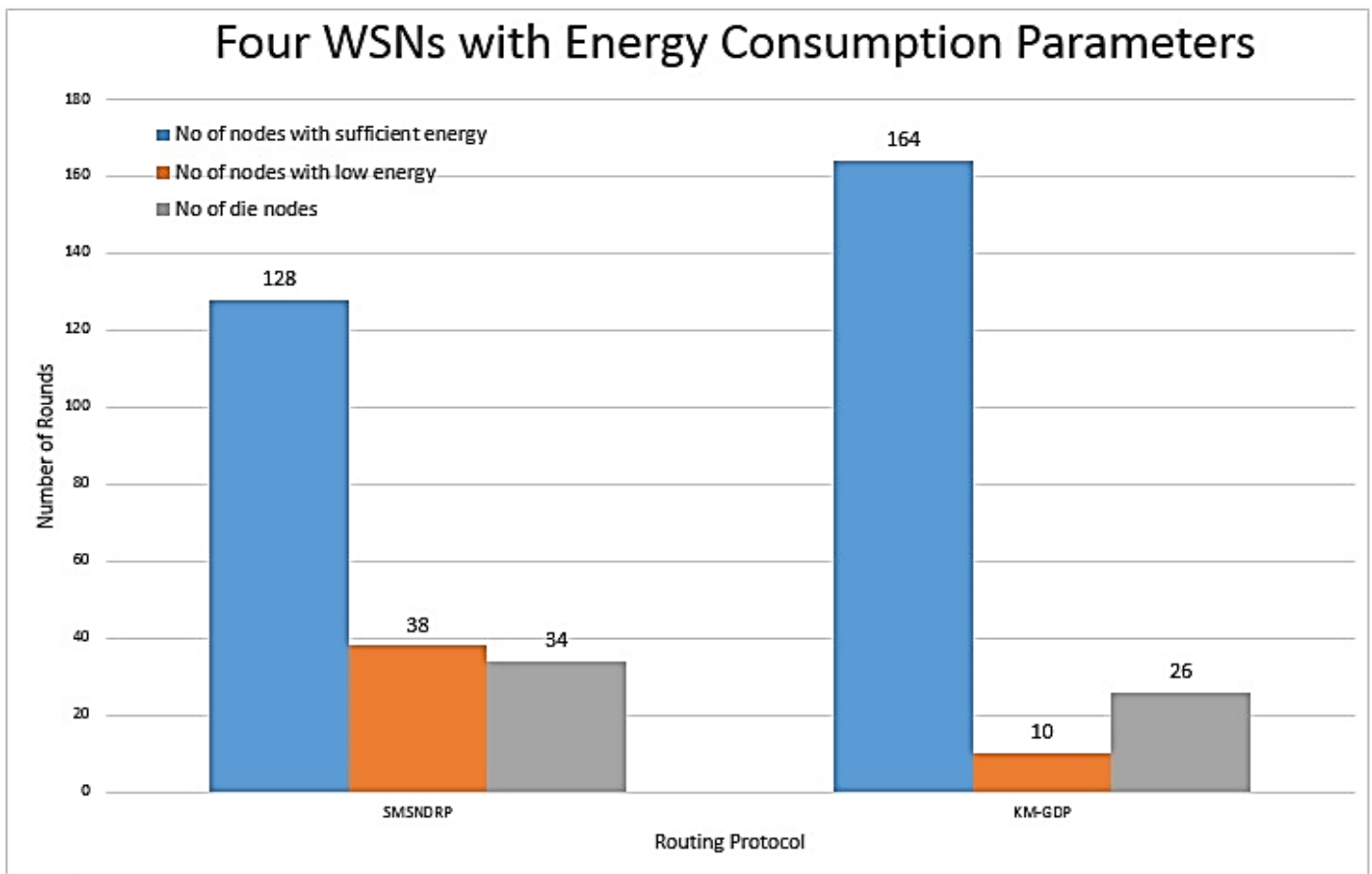

Figure 10. Demonstrate that KM-DGP given better sensor nodes' consumption in comparison with SMSNDRP

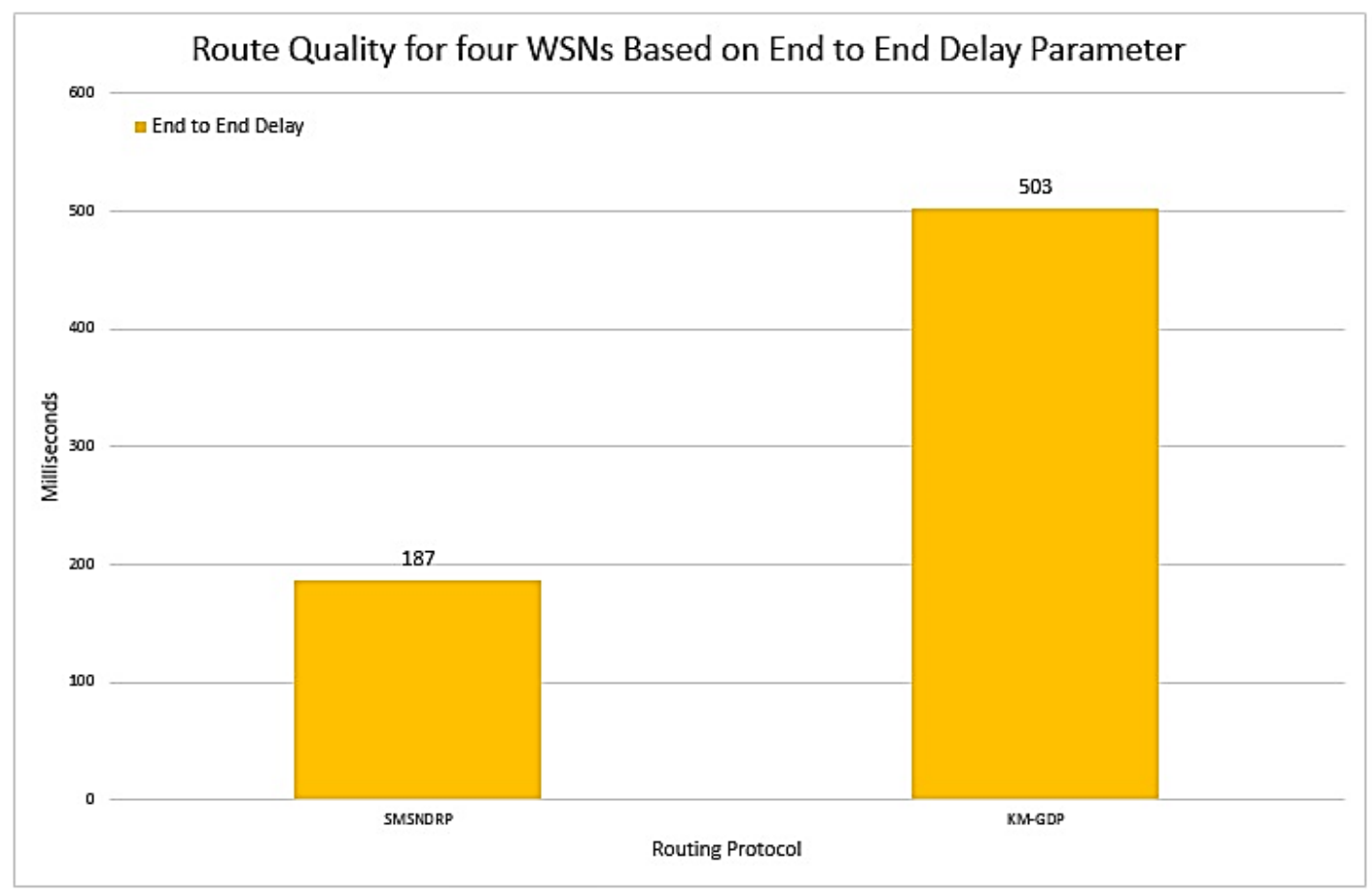

Figure 11. Demonstrate that SMSNDRP given the lowest end to end latency in comparison with KM-DGP, which make it gives better network performance than KM-DGP 


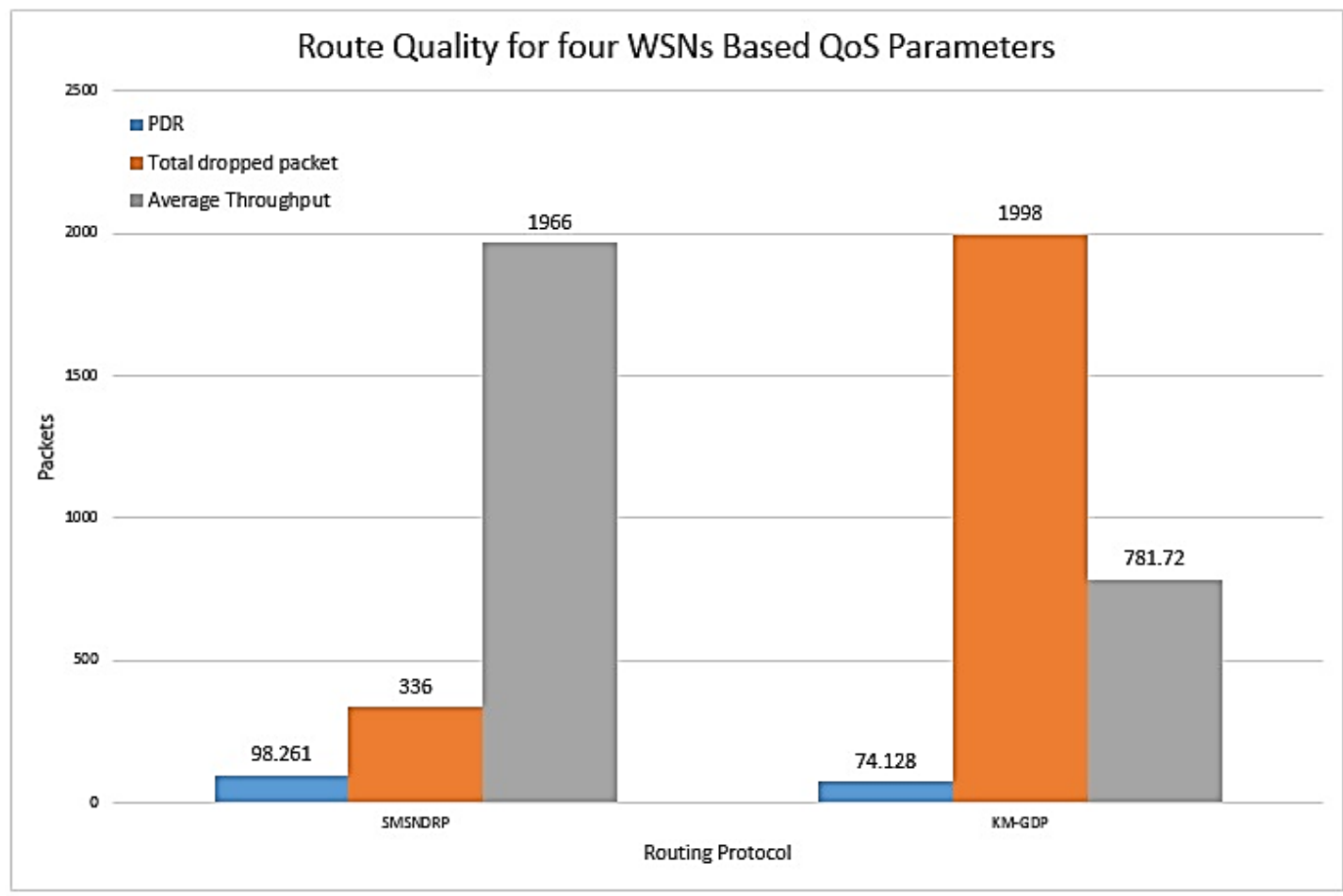

Figure 12. Demonstrate that SMSNDRP given the highest PDR, Average throughput value and lowest total dropped packets in comparison with KM-DGP, which make it gives better network performance than KM-DGP

\section{CONCLUSION}

This study is proposed an evaluation performace technique to evaluate routing protocols SMSNDRP performance in WSNs with GMSs based on QoS and sensor nodes' consumption parameters. The evaluation technique is conducted in two phases on two routing protocols SMSNDRP and KM-DGP: The first phase is used to evaluate the route quality and networks' energy consumption on small WSN with one mobile SN and GMSs. The second phase, is used to evaluate the route quality and networks' energy consumption on large network (four WSNs) with GMSs. Five sceneries are created by using NS2.3 simulator software, in order to implement the evaluation method. For the first phase three scenarios are used: the first two scenarios are conducted on one small WSN using two routing protocols (SMSNDRP and KM-DGP) with one mobile SN, the third scenario is conducted on same WSN using only routing protocol KM-DGP with four mobile SNs. While in the second phase, two scenarios are used: the first one used SMSNDRP (with four mobile SNs) and last one used KM-DGP with sixteen mobile SNs, in order to evaluate the route quality between: $(\mathrm{CH}$ and $\mathrm{SN}$ ), (node and $\mathrm{CH}$ ) and (each mobile SN in each WSN and single BS in large network).

However, it found that SMSNDRP gives better networks' energy consumption on small WSN with one mobile SN in comparison with KM-DGP with one mobile SN or four mobile SNs. Also, it generates routes that gives highest PDR value and lowest total dropped packets in contrast of using KM-DGP with one mobile SN or four mobile SNs, which make it gives better network performance in comparison with KM-DGP. But, it maximizes latency in contrast with KM-DGP. While in large network it found that KM-DGP with sixteen mobile SNs gives better network energy consumption in contrast with SMSNDRP with four mobile SNs. Nevertheless, the SMSNDRP generates a high quality route on large network with four mobile SNs in comparison of using KM-DGP with sixteen mobile SNs.

\section{ACKNOWLEDGEMENTS}

We would like to appreciate all the excellent suggestions of anonymous reviewers to enhance the quality of this paper 


\section{REFERENCES}

[1] E. Alnawafa and I. Marghescu, "New Energy Efficient Multi-Hop Routing Techniques for Wireless Sensor Networks: Static and Dynamic Techniques," Sensors, vol/issue: 18(6), pp. 1-21, 2018.

[2] T. T. Nguyen, et al., "Load balancing for mitigating hotspot problem in wireless sensor network based on enhanced diversity pollen," Journal of Information and Telecommunication, vol/issue: 2(1), pp. 91-106, 2018.

[3] Anisha P. A. and K. Raghuveer, "Load Balanced Data Delivery Using Mobile Sink in Wireless Sensor Network," International Journal of Advanced Research in Computer Science \& Technology, vol/issue: 4(2), pp. 89-91, 2016.

[4] J. John and P. Rodrigues, "Energy Efficiency Enhancement Methods for Mobile Wireless Sensor Networks: A Survey," OSR Journal of Computer Engineering (IOSR-JCE), vol/issue: 20(2), pp. 59-66, 2018.

[5] N. Ghosh and I. Banerjee, "Application of mobile sink in wireless sensor networks," 10th International Conference on Communication Systems \& Networks (COMSNETS), Bengaluru, pp. 507-509, 2018.

[6] Lee C. Y., et al., "Distributed topology control algorithm on broadcasting in wireless sensor network," J. Netw. Comput. Appl, vol. 36, pp. 1186-1195, 2013.

[7] Nazir B. and Hasbullah H., "Energy efficient and QoS aware routing protocol for Clustered Wireless Sensor Network," Comput. Electr. Eng., vol. 39, pp. 2425-244, 2013.

[8] P. Zhong and F. Ruan, "An energy efficient multiple mobile sinks based routing algorithm for wireless sensor networks," IOP Conf. Series: Materials Science and Engineering, vol/issue: 323(2018), pp. 1-5, 2018.

[9] M. Z. Abdullah, et al., "Secure Mobile Sink Node location in Wireless Sensor Network using Dynamic Routing Protocol," Association of Arab Universities Journal of Engineering Sciences, accepted published date 25/6/2018.

[10] M. Z. Abdullah, et al., "Designing Efficient Paths Between Base Station and Multi Mobile Sink Nodes to Transfer Data in Wireless Sensor Networks based on Anchor nodes," International Journal of Engineering \& Technology, vol/issue: 7(4), pp. 3810-3815, 2018.

[11] P. Chatterjee and N. Das, "Multiple sink deployment in multi-hop wireless sensor networks to enhance lifetime," 2015 Applications and Innovations in Mobile Computing (AIMoC), Kolkata, pp. 48-54, 2015.

[12] H. Barani, et al., "Effect of sink location and redundancy on multi-sink wireless sensor networks: a capacity and delay analysis," IET Communications, vol/issue: 12(8), pp. 941-947, 2018.

[13] D. R. Dandekar and P. R. Deshmukh, "Energy balancing multiple sink optimal deployment in multi-hop Wireless Sensor Networks," 3rd IEEE International Advance Computing Conference (IACC), Ghaziabad, pp. 408-412, 2013.

[14] T. K. Jain, et al., "Lifetime Optimization of a Multiple Sink Wireless Sensor Network through Energy Balancing," Journal of Sensors, vol. 2015, pp 1-6, 2015.

[15] P. Singh, et al., "An energy efficient grid based Data Dissemination routing mechanism to mobile sinks in Wireless Sensor Network," International Conference on Issues and Challenges in Intelligent Computing Techniques (ICICT), Ghaziabad, pp. 401-409, 2014.

[16] J. Wang, et al., "Bio-inspired ant colony optimization based clustering algorithm with mobile sinks for applications in consumer home automation networks," IEEE Transactions on Consumer Electronics, vol/issue: 61(4), pp. 438-444, 2015.

[17] Z. Wang, et al., "Mobile-MultiSink Routing Protocol for Underwater Wireless Sensor Networks," vol/issue: 13(6), pp. 966-974, 2017.

[18] R. S. Uppal, et al., "Reliable and energy saving multipath routing in multisink wireless sensor networks," Global Journal of Computer Science and Technology Network, Web and Security, vol/issue: 13(13), pp. 1-11, 2013.

[19] A. Gebremeskel and K. Rao, "Multiple Sink Positioning and Relocation for Improving Lifetime in Wireless Sensor Network," International Research Journal of Engineering and Technology (IRJET), vol/issue: 04(10), 2017.

[20] T. Amgoth and P. Jana, "Energy-aware routing algorithm for wireless sensor networks," Computers \& Electrical Engineering, vol. 41, pp. 357-367, 2014.

[21] I. Ha, et al., "An Optimal Data Gathering Method for Mobile Sinks in WSNs," Wireless Personal Communications, vol/issue: 97(1), pp. 1401-1417, 2017.

[22] N. A. Shiltagh, et al., "Route Quality Evolution With Mobile Sink In Wireless Sensor Networks Using QoS Parameters," Journal of Theoretical and Applied Information Technology, vol/issue: 96(23), pp. 7771-7782, 2018. 